2006

\title{
Escape rates and conditionally invariant measures
}

\author{
Mark Demers \\ Fairfield University, mdemers@fairfield.edu \\ Lai-Sang Young
}

Follow this and additional works at: https://digitalcommons.fairfield.edu/mathandcomputerscience-

facultypubs

Copyright 2006 IOP Publishing

http://iopscience.iop.org/0951-7715

\section{Peer Reviewed}

\section{Repository Citation}

Demers, Mark and Young, Lai-Sang, "Escape rates and conditionally invariant measures" (2006).

Mathematics Faculty Publications. 42.

https://digitalcommons.fairfield.edu/mathandcomputerscience-facultypubs/42

\section{Published Citation}

Mark Demers and Lai-Sang Young, "Escape rates and conditionally invariant measures," Nonlinearity, 19:2 (2006),

377-397.

This Article is brought to you for free and open access by the Mathematics Department at DigitalCommons@Fairfield. It has been accepted for inclusion in Mathematics Faculty Publications by an authorized administrator of DigitalCommons@Fairfield. For more information, please contact digitalcommons@fairfield.edu. 


\title{
Escape Rates and Conditionally Invariant Measures
}

\author{
Mark F. Demers* $\quad$ Lai-Sang Young ${ }^{\dagger}$
}

October 11, 2005

\begin{abstract}
We consider dynamical systems on domains that are not invariant under the dynamics for example, a system with a hole in the phase space - and raise issues regarding the meaning of escape rates and conditionally invariant measures. Equating observable events with sets of positive Lebesgue measure, we are led quickly to conditionally invariant measures that are absolutely continuous with respect to Lebesgue (a.c.c.i.m.). Comparisons with SRB measures are inevitable, yet there are important differences. Via informal discussions and examples, this paper seeks to clarify the ideas involved. It includes also a brief review of known results and possible directions of further work in this developing subject.
\end{abstract}

\section{Introduction}

The discussion of conditionally invariant measures in the context of dynamical systems was introduced by Pianigiani and Yorke in $[\mathrm{PY}]$ in which they posed the following question: Consider a particle on a billiard table whose dynamics are chaotic. Suppose a small hole is made in the table. What are the statistical properties of the trajectories in this system? If $p_{n}$ is the probability that a trajectory remains on the table until time $n$, what is the decay rate of $p_{n}$ ? More generally, if one starts with an initial distribution $\mu_{0}$, and $\mu_{n}$ represents the normalized distribution at time $n$ (i.e. assuming the particle has not escaped by time $n$ ), does $\mu_{n}$ converge to some $\mu$ independent of $\mu_{0}$ ?

Such a measure $\mu$, if it is well defined, is called a conditionally invariant measure for the open billiard system. There are many natural questions surrounding these measures. For example, one may consider the billiard table with a small hole as a perturbation of the billiard table with no holes, and ask if the conditionally invariant measure of the open system converges to the Liouville measure of the closed billiard system as the size of the hole tends to zero. An affirmative answer can be regarded as a form of stability of the closed system.

Dynamical systems with holes are examples of systems on domains $U$ that are not invariant under the dynamics. In the billiard example, the part of the phase space corresponding to the particle not being in the hole is our non-invariant domain of interest. Another familiar context in which such domains arise is when $U$ is a neighborhood of a non-attracting invariant set. While such invariant sets may have measure zero, it is well known that they can have nontrivial influence on global dynamics. For example, they can increase complexity, impede transport or

\footnotetext{
*Department of Mathematics, Georgia Institute of Technology. Email: demers@math.gatech.edu. This work was partially supported by NSF VIGRE grant \#DMS-0135290.

${ }^{\dagger}$ Courant Institute of Mathematical Sciences, New York University. Email: lsy@courant.nyu.edu. This work was supported by NSF Grant \#DMS-0100538
} 
slow down the rate of mixing. In much of this paper, we will not distinguish between dynamical systems with holes and dynamics on non-variant domains. Without specifying the geometry of the "hole", or equivalently the complement of $U$, the two studies are mathematically equivalent.

Although current understanding of open dynamical systems is far from complete, some progress has been made. As with closed systems, the first results are for uniformly expanding or hyperbolic systems, the simplest dynamical models that support complex phenomena. These include: expanding maps on $\mathbb{R}^{n}$ which admit a finite Markov partition after the introduction of holes [PY], [CMS1], [CMS2]; Smale horseshoes [C1],[C2]; Anosov diffeomorphisms [CM1], [CM2], [CMT1], [CMT2]; and billiards with convex scatterers satisfying a non-eclipsing condition (which eliminates some of the technical difficulties associated with billiards) [LM], [Ri]. These are followed by results on piecewise expanding maps of the interval (without Markov partitions) $[\mathrm{BK}],[\mathrm{BC}],[\mathrm{LiM}],[\mathrm{D} 1]$, and certain logistic maps [HY], [D2]. Open systems have also been studied in probabilistic settings, namely countable state Markov chains and topological Markov chains, beginning with [V] and more recently in [FKMP] and [CMS3]. Diffusion-enhanced escape rates are studied in [CMS4].

In this paper, we will focus on deterministic dynamical systems. Most of the works cited above contain detailed studies of specific systems. This paper is of a very different nature: we hope it will serve as a useful introduction to the subject. Our aim is to collect in one place basic ideas related to escape rates and conditionally invariant measures, and put them in a general, conceptual framework. We review the main results, methods and ideas that are known without getting into technical details of specific models. To this we add observations and discussions that we hope will help clarify some of the points not made explicit in previous studies. Examples are used for illustrative purposes every step of the way.

Among the points we seek to clarify is the comparison with SRB measures. Throughout this paper, we adopt the view that observable or physically relevant events are represented by positive Lebesgue measure sets. We are thus interested in conditionally invariant measures that are absolutely continuous with respect to Lebesgue (a.c.c.i.m.). Analogies with SRB measures for closed systems are both evident and inevitable, but as we will show, the situation is also quite different: unlike SRB measures, a.c.c.i.m. can often be constructed arbitrarily, with overlapping supports. This necessitates our separating those a.c.c.i.m. that are natural, in the sense that they reflect the properties of the underlying systems, from those that are not. As we will see, the meaning of "natural" is not always clear-cut, and the presence of large numbers of "unnatural" a.c.c.i.m. complicates the interpretation of results. At the same time, similarities between natural a.c.c.i.m. and SRB measures in uniformly hyperbolic systems are quite striking, and we will draw on our knowledge of SRB measures for possible insight into the properties of natural conditionally invariant measures in general.

\section{Definitions and First Examples}

In this section, we recall some basic definitions which generalize the notions introduced in the example of the billiard table.

\subsection{Basic Ideas and Questions}

\section{Setting and notation}

Consider a dynamical system defined by a self-map $\hat{T}: \hat{M} \circlearrowleft$ of a set $\hat{M}$. This paper is 
about dynamics in non-invariant regions. More precisely, we specify a subset $M \subset \hat{M}$ for which $\hat{T}(M) \cap M \neq \emptyset$ and $\hat{T}(M) \not \subset M$, and study the dynamics in $M$.

Let $H:=\hat{M} \backslash M$. We sometimes think of $H$ as a hole in the phase space, and keep track of the iterates of a point $x \in M$ as long as they do not enter $H$. Once $\hat{T}^{k} x \in H$, we say the point has fallen in the hole and no longer consider it. Alternatively, we may be interested in the dynamics near an invariant set $\Omega \subset \hat{M}$ that is not an attractor, and take $M$ to be a neighborhood of $\Omega$. Without specifying the geometry of $H$, these two problems are mathematically equivalent.

The following notation is used throughout: For $x \in M$, the smallest $n>0$ such that $\hat{T}^{n}(x) \in H$ is called its time of escape and is denoted by $E(x)$. For $n \geq 0$, we let $M^{n}:=\{x \in$ $M: E(x)>n\}$; that is to say, $M^{n}$ is the set of points that have not escaped after $n$ steps. In this notation, then, $M^{0}=M, M^{0} \supset M^{1} \supset M^{2} \supset \cdots$, and $M^{\infty}=\cap_{n \geq 0} M^{n}$.

Let $T:=\hat{T} \mid M$ denote the restriction of $\hat{T}$ to $M$. We shall refer to $T: M \rightarrow \hat{M}$ as an open system, and to $\hat{T}: \hat{M} \circlearrowleft$ as the corresponding closed system. Even though we have introduced open systems as derived from closed systems, the definition of $\hat{T}$ outside of $M$ is, in fact, immaterial to much of our discussion.

In this paper, we focus on a specific context, although parts of the discussion do not require the full force of the assumptions in this paragraph. We assume $\hat{M}$ is a compact Riemannian manifold, possibly with boundary, and $\hat{T}: \hat{M} \circlearrowleft$ is a piecewise differentiable transformation. We assume also that $H$ is open, $M$ is the closure of its interior, and that $\partial M$, the boundary of $M$, is a piecewise smooth manifold of codimension 1.

Last, but not least, we adopt in this paper the view that observable events are those having positive Lebesgue measure, by which we refer to the measure induced by Riemannian volume on $\hat{M}$. This measure is denoted by $m$ throughout.

Remark on notation: To remind ourselves that we stop considering orbits once they leave $M$, we would like to speak of iterating $T$, as opposed to $\hat{T}$. Formally, this is impossible since the range of $T$ is not contained in its domain. For notational simplicity, we will adopt the following shorthand: For $n=1,2, \cdots$, we write $T^{n}:=\left(T \mid M^{n}\right)^{n}, T_{*}^{n} \mu:=\left(T \mid M^{n}\right)_{*}^{n}\left(\mu \mid M^{n}\right)$, and so on. This formally introduces a conflict regarding whether $T=T^{1}$ is defined on $M$ or $M^{1}$, but no ambiguity will arise for as long as only image points in $M$ are considered.

\section{Rates of escape}

We begin with the most basic concept, namely the rate of escape for a given initial distribution. Let $\mu$ be a Borel probability measure on $M$. We define the upper and lower escape rates of $\mu$ to be $-\log \underline{\lambda}$ and $-\log \bar{\lambda}$ respectively where

$$
\log \bar{\lambda}:=\limsup _{n \rightarrow \infty} \frac{1}{n} \log \mu\left(M^{n}\right) \quad \text { and } \quad \log \underline{\lambda}:=\liminf _{n \rightarrow \infty} \frac{1}{n} \log \mu\left(M^{n}\right) .^{1}
$$

Here, $\bar{\lambda}=\bar{\lambda}(\mu)$ and $\underline{\lambda}=\underline{\lambda}(\mu)$. If $\underline{\lambda}=\bar{\lambda}=\lambda$, then we say the rate of escape of $\mu$ is well defined and equal to $-\log \lambda$. Clearly, $0 \leq \underline{\lambda} \leq \bar{\lambda} \leq 1$, so that given any initial distribution, the escape rate lies between 0 and $\infty$; the larger this value, the faster mass escapes from the system.

In the generality of the discussion thus far, it is obvious that $\underline{\lambda}$ and $\bar{\lambda}$ depend on $\mu$ : for the same map one chooses easily initial distributions $\mu$ so that $\mu\left(M^{n}\right)=0$ for some $n$ or $\mu\left(M^{n}\right)=1$ for all $n$. The following simple fact is useful to keep in mind: If $\mu$ and $\nu$ are two measures such that $C^{-1} \leq \frac{d \mu}{d \nu} \leq C$ for some constant $C>0$, then $\underline{\lambda}(\mu)=\underline{\lambda}(\nu)$ and $\bar{\lambda}(\mu)=\bar{\lambda}(\nu)$. We are

\footnotetext{
${ }^{1}$ We allow here the notation $\log 0=-\infty$
} 
particularly interested in Lebesgue measure as initial distribution. The upper and lower escape rates of $m$ are denoted by $-\log \underline{\Lambda}$ and $-\log \bar{\Lambda}$.

\section{Conditionally invariant probability measures}

For any Borel measure $\mu$ on $M$, let $T_{*} \mu$ denote the measure on $M$ defined by $T_{*} \mu(A)=$ $\mu\left(T^{-1}(A)\right)$ for all Borel subsets $A \subset M$. A Borel probability measure $\mu$ on $M$ is said to be conditionally invariant with respect to $T$ if

$$
\frac{T_{*} \mu(A)}{T_{*} \mu(M)}=\mu(A)
$$

for every Borel subset $A$ of $M$. The conditional invariance equation (2) says that when the measure $\mu$ loses a fraction of its mass to the hole, we renormalize by the remaining mass to recover the original measure.

Let $\mu$ be a conditionally invariant measure, and let $\lambda:=T_{*} \mu(M)=\mu\left(M^{1}\right)$. Iterating equation (2) yields $T_{*}^{n} \mu(A)=\lambda^{n} \mu(A)$ for all Borel sets $A \subset M$. In particular, $\mu\left(M^{n}\right)=$ $T_{*}^{n} \mu(M)=\lambda^{n} \mu(M)$, so $-\log \lambda$ is the escape rate defined above.

We call $\mu$ a trivial conditionally invariant measure if $\lambda=0$ or 1 . If $\lambda=0$, then we must have $\operatorname{supp}(\mu) \subseteq M \backslash M^{1}$, so that effectively all the mass falls into the hole on the first iterate. We will not be interested in such measures. If, on the other hand, $\lambda=1$, then $T(x) \in M$ for $\mu$-a.e. $x$. Such measures are essentially blind to the hole.

Observe that if $\mu$ is a nontrivial conditionally invariant measure, then for $\mu$-a.e. $x \in M$ and every $n \in \mathbb{Z}^{+}$, there exists $y \in M$ such that $T^{i}(y) \in M$ for all $i \leq n$ and $T^{n}(y)=x$, i.e., the support of $\mu$ is contained in the set of points with at least one good pre-image for all time. In particular, if $\hat{T}$ is invertible, then for $\mu$-a.e. $x \in M, \hat{T}^{-n}(x) \in M$ for all $n \geq 0$.

With regard to the existence of nontrivial conditionally invariant measures, we see that a necessary and sufficient condition is the presence of a semi-infinite orbit which stays out of the hole in backwards time, but which falls in the hole at some finite future iterate. Such an orbit is given by a sequence of points $\left\{x_{i}\right\}_{i=-\infty}^{n} \subset M$ satisfying $T\left(x_{i}\right)=x_{i+1}$ and $\hat{T}\left(x_{n}\right) \in H$. For any $0<\lambda<1$, a nontrivial conditionally invariant measure with escape rate $-\log \lambda$ is easily constructed on such an orbit by putting $\mu\left\{x_{i}\right\}=\lambda^{n-i}(1-\lambda)$ for each $i$. Since $\mu\left\{T^{-1} x_{i}\right\}=$ $\mu\left\{x_{i-1}\right\}=\lambda^{n-i+1}(1-\lambda)=\lambda \mu\left\{x_{i}\right\}$, conditional invariance follows.

Given the flexibility with which general conditionally invariant measures can be constructed - and the fact that we equate observable events with positive Lebesgue measure sets - one may wish to consider only absolutely continuous conditionally invariant measures, i.e. conditionally invariant measures that have densities with respect to $m$ (abbreviated a.c.c.i.m.). We will explain momentarily why this class alone is too restrictive for our purposes, but first we summarize the questions that have been raised:

\section{Questions raised}

- Under what conditions are escape rates well defined?

- What about the important case of $m$ as initial distribution?

- Can "reasonable" initial distributions have different escape rates?

- Do a.c.c.i.m. exist in general?

- Are they unique?

- If not, are some a.c.c.i.m. more natural than others? What does that mean?

- Must the escape rates of natural a.c.c.i.m. be compatible with that of $m$ ? 
- How are natural a.c.c.i.m. constructed?

- Can they be obtained by pushing forward Lebesgue measure and renormalizing?

Observe that in the case where $\hat{T}$ is invertible, any conditionally invariant measure is, by definition, supported on $\hat{M} \backslash\left(\cup_{n \geq 0} \hat{T}^{n}(H)\right)$. As we will see, this set often has zero Lebesgue measure. When that happens, all conditionally invariant measures are singular with respect to $m$. To the list of questions above, then, we must add the following:

- What is a natural replacement for a.c.c.i.m. for invertible maps?

In the rest of this note, we will answer these questions as best we can. As the reader will see, the picture is far from complete, and our answers will sometimes lead to further questions. This in some sense is part of the purpose of this paper.

We begin with two examples.

\section{$2.2 \quad$ First examples}

Example 1. Tent Map with a Hole. Let $\varepsilon>0$ be a small number, and let $\hat{T}:[0,1] \circlearrowleft$ be such that

$$
\hat{T}(x)=\left\{\begin{array}{ll}
\frac{2}{1-\varepsilon} x, & 0 \leq x \leq \frac{1}{2}(1-\varepsilon) \\
\frac{2}{1-\varepsilon}(1-x), & \frac{1}{2}(1-\varepsilon)<x \leq 1
\end{array} .\right.
$$

Here $H=\left(\frac{1}{2}(1-\varepsilon), \frac{1}{2}(1+\varepsilon)\right)$ is the hole. We do not specify $\hat{T}$ on $H$ since that is irrelevant. It is an easy calculation to see that normalized Lebesgue measure on $M:=[0,1] \backslash H$ is an a.c.c.i.m. for $T$ with $\lambda=1-\varepsilon$.

Example 2. Triadic Baker Map. Let $\hat{M}$ be the unit square $[0,1] \times[0,1]$ divided into three vertical strips $V_{1}, V_{2}$ and $V_{3}$ of equal width, and let $\hat{T}$ be such that $\hat{T}\left(V_{i}\right)=H_{i}$ where $H_{1}, H_{2}$ and $H_{3}$ are horizontal strips as shown. On each $V_{i}, \hat{T}$ is affine and area-preserving: $V_{i}$ is contracted by a factor of $\frac{1}{3}$ in the vertical direction and expanded by a factor of 3 in the horizontal direction.
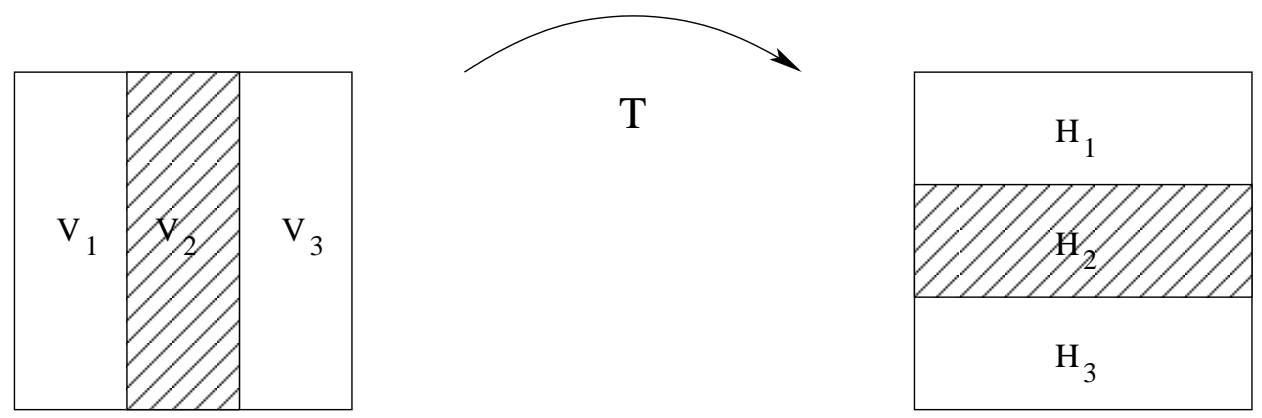

Figure 1. The triadic baker map.

Now introduce the hole $H=V_{2}$, so that $M=V_{1} \cup V_{3}$. Since $\hat{T}\left(V_{2}\right)=H_{2}$, the support of any conditionally invariant measure cannot meet this set. Similarly, it cannot meet $\hat{T}^{2}\left(V_{2}\right)$, which is the union of horizontal strips that are the middle thirds of $H_{1}$ and $H_{3}$. Continuing this line of argument, we see that any conditionally invariant measure must be supported on $\hat{M} \backslash \cup_{n \geq 0} \hat{T}^{n}\left(V_{2}\right)$, which is equal to $\left(\left[0, \frac{1}{3}\right] \cup\left[\frac{2}{3}, 1\right]\right) \times \Gamma$ where $\Gamma$ is the middle thirds Cantor set in the vertical direction. 
In this simple example, it is in fact easy to see what one obtains by pushing forward Lebesgue measure and renormalizing. Starting with $m$ on $M$, one checks that with $\Lambda=\frac{2}{3}, \Lambda^{-n} T_{*}^{n} m$ is supported on the union of $2^{n+1}$ horizontal rectangles intersected with $M$, and is uniformly distributed on it. Thus as $n$ tends to $\infty, \Lambda^{-n} T_{*}^{n} m$ converges to $\mu:=$ normalized Lebesgue measure on $\left[0, \frac{1}{3}\right] \cup\left[\frac{2}{3}, 1\right]$ crossed with the $\left(\frac{1}{2}, \frac{1}{2}\right)$-Bernoulli measure on $\Gamma$. This measure $\mu$ is, by any standard, a natural conditionally invariant measure.

Notice that $\mu$ is singular with respect to $m$ (even though $\hat{T}$ preserves $m$ ), and that it has smooth conditional measures on horizontal lines, which are unstable manifolds of $\hat{T}$.

Remark. Example 2 suggests a strong resemblance between a.c.c.i.m. in open systems and $S R B$ measures in closed systems. Here, then, is our answer to the last question in Sect. 2.1: For invertible maps, conditionally invariant measures with absolutely continuous conditional measures on unstable manifolds are natural replacements for a.c.c.i.m. To avoid cumbersome language, we use a.c.c.i.m. as an abbreviation for these measures as well.

\section{A Cautionary Note}

The two examples above paint a simple, unambiguous picture for a.c.c.i.m. and their relation to SRB measures, but unless more conditions are imposed on this class of measures, the actual situation cannot be more different. In this section we demonstrate for non-invertible maps how in quite general settings one can construct uncountably many a.c.c.i.m. with overlapping supports for any given $\lambda \in[0,1)$. Similar constructions of conditionally invariant measures smooth on unstable manifolds are easily carried out for Anosov diffeomorphisms.

\subsection{Construction of many a.c.c.i.m. with overlapping supports}

The setting we choose to illustrate our point is as follows: Continuing to use the notation in Sect. 2.1, we assume that on an open subset $V$ of $M$ of full Lebesgue measure, $T$ is locally invertible and nonsingular with respect to $m$ with Jacobian $J T>0$. We assume further that for each $x \in V, \sum_{z \in T^{-1} x}(J T(z))^{-1}<\infty$.

The idea is to identify a disjoint sequence of sets which march progressively toward the hole and eventually fall in. Additionally, these sets should consist of points with good pre-images. More precisely, for each $n \geq 1$, we let $E^{n}:=\{x \in M: E(x)=n\}$, so that $E^{n}=M^{n-1} \backslash M^{n}$. Let $G:=\left\{x \in M\right.$ : for every $n \geq 1, \exists z_{n} \in M$ such that $T^{i}\left(z_{n}\right) \in M$ for all $0 \leq i<n$ and $\left.T^{n}\left(z_{n}\right)=x\right\}$.

Theorem 3.1 Let $(\hat{T}, \hat{M}, H)$ be as above. We assume

$$
m\left(E^{1} \cap G\right)>0 .
$$

Then given any $\lambda, 0 \leq \lambda<1, T$ admits uncountably many a.c.c.i.m. with escape rate $-\log \lambda$.

Proof. Let $G^{n}:=E^{n} \cap G$. We observe that

(i) for $n=1,2, \ldots$, the sets $G^{n}$ are pairwise disjoint with $m\left(G^{n}\right)>0$;

(ii) for each $n>1, T\left(G^{n}\right)=G^{n-1}$ and $T^{-1}\left(G^{n-1}\right) \cap G=G^{n}$.

That $T\left(G^{n}\right) \subset G^{n-1}$ is obvious. Consider $x \in G^{n-1}$. Since $x \in G$, there exists a sequence of pre-images $\left\{z_{i}\right\}_{i=1}^{\infty}$ associated with $x$ such that $T\left(z_{i}\right)=z_{i-1}$ and $T^{i}\left(z_{i}\right)=x$ for all $i$. Note that 
each $z_{i} \in G$ as well using the same sequence. In particular, $z_{1} \in G^{n}$. This proves (ii). As for (i), the sets $G^{n}$ are pairwise disjoint because the $E^{n}$ are, and the fact that they have positive measure follows inductively from $m\left(G^{1}\right)>0, T\left(G^{n}\right)=G^{n-1}$ and $J T>0$.

Fix $\lambda$ such that $0 \leq \lambda<1$, and let $\psi \geq 0$ be an integrable function with $\int_{G^{1}} \psi d m=1$. On $G^{1}$, define

$$
f(x)=(1-\lambda) \psi(x) .
$$

Note that $\int_{G^{1}} f d m=1-\lambda$. This is the amount that falls in the hole at time 1.

Proceeding inductively, suppose $f$ has been defined on $G^{n-1}$. For $y \in G^{n}$, we let $T(y)=x$, and define

$$
f(y)=\frac{\lambda f(x)}{\sum_{z \in T^{-1} x \cap G} \frac{1}{J T(z)}}
$$

so that $f$ is constant on $T^{-1} x \cap G$. Set $f=0$ on $\hat{M} \backslash \cup_{n} G^{n}$.

To prove conditional invariance with escape rate $-\log \lambda$, it suffices, in light of (i), (ii) and the definition of $f$ on $G^{1}$, to check that for each $n>1$,

$$
\mathcal{P}_{\hat{T}}\left(f \mid G^{n}\right)=\lambda f \mid G^{n-1}
$$

where $\mathcal{P}_{\hat{T}}$ is the usual Perron-Frobenius operator associated with $\hat{T}$. This is true by the way we have defined $f$ in equation (3). Finally, we check that

$$
\int_{\hat{M}} f d m=\sum_{n=1}^{\infty} \int_{G^{n}} f d m=\sum_{n=1}^{\infty} \lambda^{n-1} \int_{G^{1}} f d m=\sum_{n=0}^{\infty} \lambda^{n}(1-\lambda)=1 .
$$

Since the choice of $\psi$ is entirely arbitrary, the construction above gives uncountably many a.c.c.i.m. as desired.

Remark. As the proof above shows, a.c.c.i.m. can be constructed quite arbitrarily - with overlapping supports if one so desires - once a suitable sequence of sets in $M$ is located. Given arbitrary $\bar{\lambda} \geq \underline{\lambda}$, similar constructions give absolutely continuous initial distributions with $-\log \underline{\lambda}$ and $-\log \bar{\lambda}$ as their upper and lower escape rates.

We conclude that absolute continuity with respect to $m$ alone is not a sufficient condition for identifying a meaningful class of conditionally invariant measures.

\subsection{Examples}

We revisit Example 1 in Sect. 2.2, the tent map with a hole. Observe that in this example, $G=[0,1] \backslash H$, and for each $n \geq 1, G^{n}=E^{n}$ is the union of $2^{n}$ disjoint intervals of length $\left(\frac{1-\varepsilon}{2}\right)^{n}$ each. The following are three examples of a.c.c.i.m. obtained from the constructions in the proof of Theorem 3.1:

(i) $\lambda=1-\varepsilon$ and $\psi=\frac{1}{m\left(E^{1}\right)}$. The construction in Theorem 3.1 gives $\mu=$ normalized Lebesgue measure on $[0,1] \backslash H$. It is easy to check that $\lambda=\Lambda$ where $-\log \Lambda$ is the escape rate of Lebesgue measure. 
(ii) Let $E^{1}=:\left[a_{1}, b_{1}\right] \cup\left[a_{2}, b_{2}\right]$. On $\left[a_{1}, b_{1}\right]$, we define $\psi(x)=\frac{1}{m\left(E^{1}\right)}\left(1+c \sin \frac{2 \pi\left(x-a_{1}\right)}{b_{1}-a_{1}}\right) ; \psi$ is defined analogously on $\left[a_{2}, b_{2}\right]$. For suitable choice of $c$, the construction in Theorem 3.1 yields an a.c.c.i.m. with density bounded away from zero and infinity, so it follows that $\lambda=\Lambda$. The density, however, is not of bounded variation. ${ }^{2}$

(iii) Choose $\alpha<\frac{1}{2}$ and set $f=\alpha^{n}$ on $E^{n}$ for $n \geq 1$. The resulting conditionally invariant measure has a density of bounded variation and $\lambda=\alpha(1-\varepsilon)$ so that its escape rate is strictly greater than $-\log \Lambda$. (This construction is due to Chernov and van den Bedem; see $[\mathrm{BC}]$.)

\section{Rates of Escape}

In light of Theorem 3.1, we assume in this section that Lebesgue measure, or measures equivalent to it with densities bounded away from zero and infinity, are the only natural initial distributions, and review some basic facts about escape rates. This point of view may be overly simplistic (see the Remark at the end of Sect. 4.2), but we will adopt it for purposes of the present discussion. We focus in this section on escape rates only; conditionally invariant measures are discussed in Section 5 .

The uniform hyperbolic picture is, as usual, a model of good behavior. A result for these systems and its partial generalization are given in Sect. 4.1. Examples with super-exponential and sub-exponential escape rates are discussed in Sect. 4.2.

\subsection{Relation to Lyapunov Exponents and Entropy}

Let $X$ be a compact metric space, $\hat{T}: X \circlearrowleft$ a continuous map, and $\varphi: X \rightarrow \mathbb{R}$ a continuous function. Recall that the topological pressure of $\hat{T}$ for the potential $\varphi$ is defined to be

$$
P(\hat{T}, \varphi):=\sup \left\{h_{\nu}(\hat{T})+\int \varphi d \nu\right\}
$$

where the supremum above is taken over all $\hat{T}$-invariant Borel probability measures $\nu$ on $X$ and $h_{\nu}(\hat{T})$ is the metric entropy of $\hat{T}$ with respect to the measure $\nu$. A measure that realizes the supremum in (4) is called an equilibrium state of $\hat{T}$ for the potential $\varphi$.

Consider now a compact invariant set $\Omega$ of a diffeomorphism $\hat{T}$ with the property that $\hat{T} \mid \Omega$ is uniformly hyperbolic, and let $M$ be a small neighborhood of $\Omega$. We assume $\Omega$ is the maximal invariant set in $M$, i.e., $\Omega=\cap_{n \in \mathbb{Z}} \hat{T}^{n}(M)$. It is proved in [B] and [Y1] that the escape rate $-\log \Lambda$ from $M$ is well defined with

$$
\log \Lambda=P(\hat{T}|\Omega, \varphi| \Omega), \quad \varphi=-\log \left|\operatorname{det}\left(\left.D \hat{T}\right|_{E^{u}}\right)\right| .
$$

Moreover, $0<\Lambda \leq 1 ; \Lambda=1$ if and only if $\Omega$ is an attractor.

In general, without any assumptions on $\hat{T}$ other than $C^{2}$, we continue to have

$$
\log \underline{\Lambda} \geq \sup \left\{h_{\nu}(\hat{T})-\sum \chi_{i}^{+}(\nu)\right\}
$$

\footnotetext{
${ }^{2}$ We mention the idea of bounded variation because it is well known that densities in this class are preserved by piecewise expanding maps (without holes). The connection will become clear in Section 5 .
} 
Here $\nu$ ranges over all ergodic Borel probability measures of $\hat{T}$, and $\chi_{i}^{+}(\nu)$ are the positive Lyapunov exponents with respect to $\nu$ counted with multiplicity [Y1]. The idea of the proof is as follows: Let

$$
V(x, n, \varepsilon):=\left\{y \in M: d\left(\hat{T}^{i} x, \hat{T}^{i} y\right)<\varepsilon \text { for } 0 \leq \mathrm{i}<\mathrm{n}\right\} .
$$

A general fact proved in [Y1] says that for $\nu$-typical $x, m(V(x, n, \varepsilon)) \lesssim e^{-n \sum \chi_{i}^{+}(\nu)}$. The bound in (6) then follows from the fact that $M^{n}$ contains $\sim e^{n h_{\nu}}$ disjoint $V(x, n, \varepsilon)$.

The reverse inequality to (6) is not valid without some additional hypotheses. The simplest example in which the inequality fails is when $M$ is a neighborhood of the so-called Figure- 8 attractor: Since $\Omega$ is an attractor, the escape rate is zero. On the other hand, Dirac measure at the saddle fixed point is the only invariant measure, so the right side of (6) is strictly negative. For another example, see [BBS].

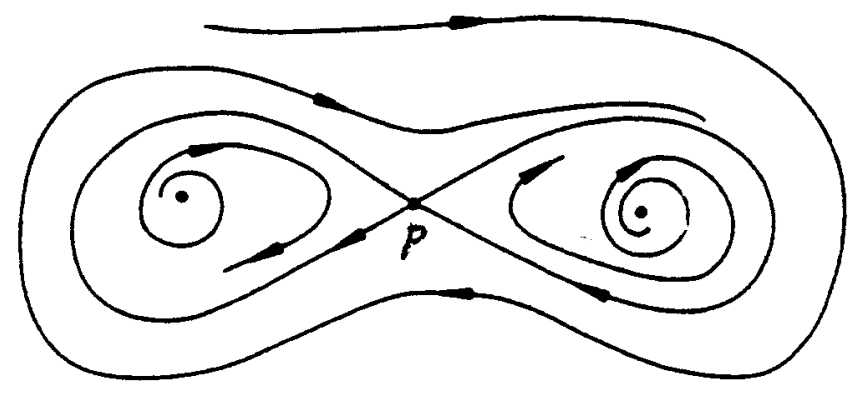

Figure 2. The figure- 8 attractor.

\subsection{Escape rates that are sub-exponential or super-exponential}

In this subsection we consider some situations where Lebesgue measure escapes at superexponential rates (corresponding to $\Lambda=0$ ) and at sub-exponential rates (corresponding to $\Lambda=1)$.

Our first example illustrates how unbounded derivatives may lead to super-exponential escape rates. While this example is very simple and somewhat artificial, unbounded derivatives do occur naturally in physical systems modeling the motion of particles or objects with collisions.

Example 3. An expanding map with unbounded derivative. Consider the map $\hat{T}$ : $[0,1] \circlearrowleft$ defined by

$$
\hat{T}(x)=\left\{\begin{array}{ll}
\sqrt{2 x}, & 0 \leq x \leq \frac{1}{2} \\
2\left(x-\frac{1}{2}\right), & \frac{1}{2}<x \leq 1
\end{array} .\right.
$$

Let $M=\left[0, \frac{1}{2}\right]$ and note that both $\hat{T}$ and $T$ admit a finite Markov partition. From Theorem 3.1, we know that this system has many a.c.c.i.m.s with any escape rate $0 \leq \lambda<1$. We claim that none of them is a good indicator for what happens to Lebesgue measure. A simple calculation gives $m\left(M^{n}\right)=2 \cdot 2^{-2^{n+1}}$, so that Lebesgue measure escapes at a super-exponential rate.

Example 4 below describes a simple situation in which Lebesgue measure escapes at a subexponential rate. 
Example 4. An expanding map with a neutral fixed point. Let $\hat{T}:[0,1] \circlearrowleft$ be defined as follows:

$$
\hat{T}(x)=\left\{\begin{array}{ll}
x+\sqrt{2} x^{3 / 2}, & 0 \leq x \leq \frac{1}{2} \\
2 x-1, & \frac{1}{2}<x \leq 1
\end{array} .\right.
$$

The only restriction we put on $H$ is that $M$ should contain a neighborhood of the origin. We will show that $m\left(M^{n}\right) \geq \frac{c}{n^{2}}$ for all sufficiently large $n$. Let $\psi$ denote the inverse of $\hat{T}$ on $\left[0, \frac{1}{2}\right]$. Define $x_{1}=\psi\left(\frac{1}{2}\right)$ and in general, let $x_{n}=\psi\left(x_{n-1}\right)=\psi^{n}\left(\frac{1}{2}\right)$ for each $n>0$. We observe that $x_{n}$ has the same asymptotics as the sequence $\left\{\frac{1}{k^{2}}\right\}$ : Let $\Delta_{n}=x_{n}-x_{n+1}$. Then $\Delta_{n}=T\left(x_{n+1}\right)-x_{n+1}=\sqrt{2}\left(x_{n+1}\right)^{3 / 2}$, whereas $\frac{1}{n^{2}}-\frac{1}{(n+1)^{2}} \sim \frac{1}{n^{3}}=\left(\frac{1}{n^{2}}\right)^{3 / 2}$. It follows that if $x_{n} \in\left[\frac{1}{(k+1)^{2}}, \frac{1}{k^{2}}\right]$, then there is a uniform bound on the number of intervals $\left[\frac{1}{(k+1)^{2}}, \frac{1}{k^{2}}\right]$ which intersect $\left[x_{n+1}, x_{n}\right]$ and vice versa. Since $M$ contains a neighborhood of the origin, there exists an $n_{0} \geq 0$ such that $\left[x_{k}, x_{k-1}\right] \subset M$ for all $k \geq n_{0}$. It follows that $\left[x_{k+n_{0}}, x_{k-1+n_{0}}\right]$ does not fall in the hole until at least time $k$, so that $\left[x_{k+n_{0}}, x_{k-1+n_{0}}\right] \subset M^{k-1} \backslash M^{k}$. We conclude that $m\left(M^{k-1}\right) \geq \frac{c}{\left(k+n_{0}\right)^{2}}$.

Our next example consists of a class of objects sometimes referred to as Markov towers. These objects were introduced in [Y2] and [Y3]. They appear naturally as Markov extensions of many nonuniformly hyperbolic systems, generalizing the idea of Markov partitions (see e.g. $[\mathrm{CY}])$. The structure of a tower allows for "tails" in return times with various asymptotics which have been shown to carry much of the statistical information of the system. For example, return times $R$ with $m\{R>n\} \sim \frac{1}{n^{\alpha}}$ correspond to polynomial rates of correlation decay.

Example 5. Markov towers: tail bounds and escape rates. We go directly to the tower map, which we denote by $\hat{T}: \hat{M} \circlearrowleft$, ignoring the dynamical system of which this tower is intended to be a Markov extension. Roughly speaking, $\hat{M}$ has the structure

$$
\hat{M}=\left\{(x, n) \in \hat{\Delta}_{0} \times \mathbb{N}: R(x)>n\right\}
$$

where $\hat{\Delta}_{0} \subset \hat{M}$ is a set with $m\left(\hat{\Delta}_{0}\right)>0$ and $R$ is a return time function to the set $\hat{\Delta}_{0}$. The set $\hat{\Delta}_{\ell}:=\left.\hat{\Delta}\right|_{n=\ell}$ is called the $\ell^{\text {th }}$ level of the tower. The tower map $\hat{T}$ is given by

$$
\hat{T}=\left\{\begin{array}{ll}
\hat{T}(x, n)=(x, n+1) & \text { if } n+1<R(x) \\
\hat{T}(x, n)=\left(T^{R}(x), 0\right) & \text { if } n+1=R(x)
\end{array} .\right.
$$

That is to say, the action of the tower map $\hat{T}$ is to map a point up the levels of the tower until time $R$ at which time the point is returned to the base $\hat{\Delta}_{0}$. The definition of $\hat{T}$ implies that for every $\ell \geq 0, \hat{T}$ carries $m$ on $\hat{\Delta}_{\ell} \cap \hat{T}^{-1} \hat{\Delta}_{\ell+1}$ to $m$ on $\hat{\Delta}_{\ell+1}$, i.e. Lebesgue measure is preserved as one goes up the tower. (Notice that no such assumption is made about the map that takes the top level of the tower back to $\hat{\Delta}_{0}$.) On $\hat{\Delta}_{0}$ there is a countable partition $\mathcal{Z}$ with the following properties: $R$ is constant on each element $\omega \in \mathcal{Z}$, and $T^{R(\omega)}$ maps $\omega$ bijectively onto $\hat{\Delta}_{0}$. For more detail, see $[Y 3]$.

We introduce holes into the tower as follows. For simplicity, we consider only Markov holes, i.e. $H_{\ell}$ on the $\ell^{\text {th }}$ level of the tower is the union of the $\hat{T}^{\ell}$-images of a finite number of elements of $\mathcal{Z}$. If $\hat{T}^{\ell} \omega \subseteq H_{\ell}$, we also throw out all sets of the form $\hat{T}^{k} \omega$ for $k>\ell$ since nothing will be mapped there once the hole is introduced. We let $\Delta_{\ell}$ denote the $\ell^{\text {th }}$ level of the tower after these deletions and let $M=\cup \Delta_{\ell}$. 
Consider the case where $m \Delta_{\ell} \sim \ell^{-\alpha}$ for some $\alpha>1$. Since $T^{-n} \Delta_{n} \subset \Delta_{0}$ and $T$ preserves Lebesgue measure mapping up the levels of the tower, one sees that $m\left(M^{n}\right) \geq m\left(\hat{T}^{-n} \Delta_{n}\right)=$ $m \Delta_{n} \sim n^{-\alpha}$ so that Lebesgue measure escapes at a polynomial rate.

If, on the other hand, we have a tower with exponentially decreasing tails, $m \Delta_{\ell} \sim \theta^{\ell}$ for some $\theta<1$, then for sufficiently small holes, it is shown in [D1] that an a.c.c.i.m. exists with density bounded away from zero on $M$. This implies the exponential escape rate of Lebesgue measure.

Remark on initial distributions. We have, for simplicity, assumed in the discussion above that the only natural initial distributions $\mu_{0}$ are measures equivalent to $m$; that is likely to be an over-simplification. For example, it is quite natural to take $\mu_{0}$ to be an SRB measure of the corresponding closed system, the rationale being that if the "leak" starts only after the system has been running for some time, then the measure that best describes its state at the start of the leak would be its SRB measure. Finally, in open systems that are in contact with larger systems ("heat reservoirs"), $\mu_{0}$ tends to belong to certain specific classes. It is probably prudent to retain a certain amount of flexibility without viewing all initial distributions as equal.

\section{Conditionally Invariant Measures}

In this section we review three types of methods for constructing or proving the existence of a.c.c.i.m., and summarize the results obtained thus far. The first method relies on the linear structure of conditional transfer operators; the second seeks to show that normalized pushed-forward densities lie in certain compact sets; and the third is by introducing Markov structures. All three follow closely standard techniques for constructing SRB measures. A new consideration not present in discussions of SRB measures is the degree to which the measures constructed reflect the properties of the systems in question. It may be useful to keep in mind the cautionary note in Sect. 3.1 while reading this section.

\section{$5.1 \quad$ Interpretation of results}

Before embarking on a discussion of methods of construction, we first describe what we hope to achieve. Define the normalized operator

$$
\mathcal{T}_{1} \mu=\frac{T_{*} \mu}{\left|T_{*} \mu\right|}
$$

where $\left|T_{*} \mu\right|=T_{*} \mu(M)$ and $\mu$ is any Borel probability measure for which $\left|T_{*} \mu\right| \neq 0$.

The ideal situation. The best scenario is one in which for some Borel measure $\mu$,

$$
\mathcal{T}_{1}^{n} m \rightarrow \mu \quad \text { as } n \rightarrow \infty
$$

where convergence is at least in the weak* topology. If (7) holds and $\mu$ is a point of continuity ${ }^{3}$ for $\mathcal{T}_{1}$ acting on a suitable set of measures including $m$, then: (i) $\left|T_{*} \mu\right|=\Lambda$, the escape rate of Lebesgue measure; (ii) $T_{*} \mu=\Lambda \mu$, and $\mu$ is the conditionally invariant measure of interest.

Remarks. We would like to think of $\mu$ defined by (7) as our natural a.c.c.i.m. but there are two complications:

\footnotetext{
${ }^{3}$ See $[\mathrm{BB}]$ for possible anomalies if this condition is not met.
} 
1. $\mu$ obtained from (7) may not be an a.c.c.i.m.. Indeed, there are situations for which (7) holds but no natural a.c.ci.m. exists. (See Example 4, where $\mathcal{T}_{1}^{n} m$ converges to the Dirac measure at the fixed point 0.) Whether or not the limit $\mu$ in (7) has a density, it gives a good indication of what the distributions $\mathcal{T}_{1}^{n} m$ for large $n$ look like, and that is important in practical situations. Where $\mu$ is not an a.c.c.i.m., however, the open system $(T, \mu)$ by itself may not be especially enlightening.

2. One may wish to strengthen (7) to require that $\mathcal{T}_{1}^{n} \mu_{0} \rightarrow \mu$ for a reasonably large class of $\mu_{0}$, such as those with densities. From Sect. 3.1, we know that this would not make sense in general. Indeed Sects. 5.2, 5.3 and 6 demonstrate quite clearly that in general, one must suitably limit the class of allowed initial distributions $\mu_{0}$ - in a way that depends on the characteristics of the system - or there can be no common limit measure $\mu$. A model-dependent notion of "naturalness" for a.c.c.i.m. is not satisfactory, but that, unfortunately, is the state of affairs.

The next best case. When we are unable to prove the convergence of $T_{1}^{n} m$, or when $T_{1}^{n} m$ does not converge, the next best scenario is to have some type of convergence on average. Examples of relevant types of averages are

$$
\frac{1}{n} \sum_{i<n} \frac{T_{*}^{i} m}{\left|T_{*}^{i} m\right|} \quad \text { or } \quad \frac{1}{n} \sum_{i<n} \lambda^{-i} T_{*}^{i} m \quad \text { or } \quad \frac{\sum_{i<n} \lambda^{-i} T_{*}^{i} m}{\sum_{i<n} \lambda^{-i}\left|T_{*}^{i} m\right|} .
$$

The limit measures $\mu$ so obtained are fairly natural, and it is not unreasonable to think of their escape rates as the average escape rates of Lebesgue measure. (The conditional invariance of $\mu$, however, is not always transparent.) Without requiring convergence, one may consider accumulation points of the sequences above. Needless to say, the weaker the condition satisfied, the more tenuous the claim that the resulting objects represent the true escape dynamics of the system.

Other possibilities. Suppose we are not able to prove (7) directly, but find by some other means an a.c.c.i.m. $\mu$ with $T_{*} \mu=\lambda \mu$ and $\frac{d \mu}{d m}=f$. Then provided that $C^{-1} \leq f \leq C$ for some constant $C$, we may conclude that $\lambda=\Lambda$ (see Sect. 2.1), and if $f$ is only bounded away from zero or infinity, but not both, then one of the inequalities remains valid (for example, if we know only that $f \geq C^{-1}$, then $\lambda \geq \bar{\Lambda}$ ). Bounding the density above and below can sometimes be used to prove uniqueness in a certain class of functions (see Sect. 5.3). See also the last Remark at the end of Section 4.

One final point: Recall that there are two kinds of a.c.c.i.m.: the first type of conditionally invariant measure is absolutely continuous with respect to $m$, while the second is smooth only on unstable manifolds. For simplicity we will focus mostly on the first kind, noting that all additional technical difficulties in going from invariant densities to SRB measures in closed systems appear also in the treatment of a.c.c.i.m. of the second kind.

\subsection{Conditional transfer operators and eigenvalue problems}

In situations where $\hat{T}$ has invariant densities, (7) is often deduced from transfer operator arguments. More precisely, assume $\hat{T}$ is locally invertible with Jacobian $J \hat{T}$. The usual PerronFrobenius or transfer operator associated with $\hat{T}$ is defined by

$$
\mathcal{P}_{\hat{T}} f(x):=\sum_{y \in \hat{T}^{-1} x} \frac{f(y)}{|J \hat{T}(y)|} .
$$


Using $T$ instead of $\hat{T}$, and letting both $f$ and $\mathcal{P}_{T} f$ be functions defined on $M$, we have the conditional transfer operator

$$
\mathcal{P}_{T} f(x):=\sum_{y \in T^{-1} x} \frac{f(y)}{|J T(y)|} .
$$

That is to say,

$$
\mathcal{P}_{T} f=\mathcal{P}_{\hat{T}}\left(\hat{f} \cdot 1_{M^{1}}\right)
$$

where $\hat{f}$ is any extension of $f$ to $\hat{M}$. Observe that the operator $\mathcal{P}_{T}$, which we will call $\mathcal{P}$ from here on, satisfies the usual iterative relation $\mathcal{P}_{T^{n}}=\mathcal{P}_{T}^{n}$. We define the normalized operator $\mathcal{P}_{1}$ by

$$
\mathcal{P}_{1} f=\frac{\mathcal{P} f}{|\mathcal{P} f|_{L^{1}(M)}}
$$

and note that if $f$ is the density corresponding to a measure $\mu$, then $\mathcal{P}_{1} f$ is the density corresponding to $\mathcal{T}_{1} \mu$.

\section{Spectral properties of $\mathcal{P}$}

This method is a direct generalization of the corresponding analysis for $\mathcal{P}_{\hat{T}}$.

Suppose that with $\mathcal{P}$ acting on a Banach space $(\mathcal{B},\|\cdot\|)$ with $\mathcal{B} \subset L^{1}(M)$, one finds an eigenvalue $\lambda \in(0,1]$ with eigenfunction $f \geq 0$. Let $f$ be such that $\int f d m=1$. It follows immediately that $T_{*} \mu=\lambda \mu$ where $\mu$ is the measure with $\frac{d \mu}{d m}=f$.

If the operator $\mathcal{P}$ is quasi-compact and $\lambda$ has multiplicity one and is the unique eigenvalue of maximum modulus, then the linear structure of $\mathcal{P}$ implies the following: (i) $\mathcal{B}=\mathcal{H} \oplus\{f\}$ where $\mathcal{P}(\mathcal{H}) \subset \mathcal{H}$ and $\{f\}$ denotes the 1-dimensional space spanned by $f$, and (ii) for all $g \in \mathcal{B} \backslash \mathcal{H}$, there exists $c(g) \neq 0$ such that $\left\|\lambda^{-n} \mathcal{P}^{n} g-c(g) f\right\| \rightarrow 0$; equivalently, $\left\|\mathcal{P}_{1}^{n} g-f\right\| \rightarrow 0$. If furthermore there exists $g \in \mathcal{B} \backslash \mathcal{H}$ such that $g$ is bounded away from zero and infinity, then $\lambda=\Lambda$ where $-\log \Lambda$ is the escape rate of $m$, and $\mathcal{T}_{1}^{n} m \rightarrow \mu$. This is the ideal case.

For a system without holes, eigenfunctions $h$ of $\mathcal{P}_{\hat{T}}$ associated with eigenvalues $\alpha \neq 1$ cannot give rise to probability measures due to the identity

$$
\alpha \int_{\hat{M}} h d m=\int_{\hat{M}} \mathcal{P}_{\hat{T}} h d m=\int_{\hat{M}} h d m
$$

which implies that $h$ cannot be nonnegative. By contrast, once a hole is introduced into the system, the second equality above becomes an inequality. In general we expect to find many positive eigenfunctions in $\mathcal{B}$ (see the example of Sect. 3.2). In the setting of the last paragraph, since $\alpha<\lambda$, we have $\lambda^{-n} \mathcal{P}^{n} h \rightarrow 0$, so that all conditionally invariant densities $h \in \mathcal{B}$ other than $f$ lie in $\mathcal{H}$ and have escape rates greater than $-\log \lambda$.

The quasi-compactness of the transfer operator has been proven for piecewise expanding maps of the interval with holes in $[\mathrm{LiM}]$; see Section 6.

\section{Projective Cones}

A second method uses the Hilbert or projective metric due to G. Birkhoff [Bi]. We review the basic setup: Given a vector space $\mathcal{V}$, a convex subset $\mathcal{C} \subset \mathcal{V}$ is called a cone if: (i) $\mathcal{C} \cap-\mathcal{C}=\emptyset$; (ii) $\alpha \mathcal{C}=\mathcal{C}$ for all $\alpha>0$; (iii) for all $f, g \in \mathcal{C}$, if $\alpha_{n} \in \mathbb{R}, \alpha_{n} \rightarrow \alpha$, and $g-\alpha_{n} f \in \mathcal{C}$ for each $n$, then $g-\alpha f \in \mathcal{C} \cup\{0\}$. The Hilbert metric on $\mathcal{C}$ is defined by

$$
d(f, g)=\log \frac{\beta(f, g)}{\alpha(f, g)}
$$


where $\alpha(f, g)=\sup \{a>0 \mid g-a f \in \mathcal{C}\}$ and $\beta(f, g)=\inf \{b>0 \mid b f-g \in \mathcal{C}\}$. Now let $\mathcal{L}: \mathcal{V} \rightarrow \mathcal{V}$ be a linear operator, and assume $\mathcal{L}(\mathcal{C}) \subset \mathcal{C}$. We define the diameter of $\mathcal{L}(\mathcal{C})$ to be $\Delta:=\sup _{f, g \in \mathcal{C}} d(\mathcal{L} f, \mathcal{L} g) \leq \infty$. A result of $[\mathrm{Bi}]$ implies that for all $f, g \in \mathcal{C}, d(\mathcal{L} f, \mathcal{L} g) \leq$ $\tanh \left(\frac{\Delta}{4}\right) d(f, g)$. In particular, this means that if $\Delta<\infty$, then $\mathcal{L}$ has a uniform rate of contraction $\alpha=\tanh \left(\frac{\Delta}{4}\right)<1$ in the Hilbert metric.

Returning to our setting, if $\mathcal{P}=\mathcal{L}$ meets the conditions above with respect to appropriate $\mathcal{V}$ and $\mathcal{C}$, then we may conclude the existence of a unique conditionally invariant density $f \in \mathcal{C}$ with escape rate $\lambda=\int \mathcal{P} f d m$. Let $\mu$ be the a.c.c.i.m. with density $f$.

Proving that $\mathcal{T}_{1}^{n} \nu \rightarrow \mu$ where $\nu$ has density in $\mathcal{C}$ requires an extra argument. Notice first that the contraction above is in the Hilbert metric. Following [L], this contraction can be passed to norms $|\cdot|$ on $\mathcal{V}$ which are adapted to $\mathcal{C}$ (i.e. for all $g, h \in \mathcal{C}, h-g \in \mathcal{C}$ and $h+g \in \mathcal{C}$ implies $|h| \geq|g|)$ via

$$
|g-h| \leq\left(e^{d(g, h)}-1\right)|g| \quad \text { for all } g, h \in \mathcal{C} \text { with }|h|=|g| .
$$

In our setting, if for example the $L^{1}$ norm is adapted, then iterating $\mathcal{P}$ and noting that $\left|\mathcal{P}_{1}^{n} g\right|_{1}=$ $\left|\mathcal{P}_{1}^{n} f\right|_{1}=1$ (even though the corresponding statement is false for $\mathcal{P}$ ), we obtain

$$
\begin{aligned}
\left|\mathcal{P}_{1}^{n} g-f\right|_{1} & =\left|\mathcal{P}_{1}^{n} g-\mathcal{P}_{1}^{n} f\right|_{1} \leq e^{d\left(\mathcal{P}_{1}^{n} g, \mathcal{P}_{1}^{n} f\right)}-1 \\
& =e^{d\left(\mathcal{P}^{n} g, \mathcal{P}^{n} f\right)}-1 \leq e^{\alpha^{n} d(f, g)}-1
\end{aligned}
$$

We have used in the second line that $d$ is a projective metric and so invariant under scalar multiples. This implies that $\mathcal{P}_{1}^{n} g \rightarrow f$ in $L^{1}$ for all $g \in \mathcal{C}$ at a uniform exponential rate. As before, if $1 \in \mathcal{C}$ or if there exists any $g \in \mathcal{C}$ which is bounded away from zero and infinity, then $\lambda=\Lambda$. This again is the best case scenario.

This method was introduced for closed systems in $[\mathrm{FS}]$ and $[\mathrm{L}]$. For open systems, it was used for 1D piecewise expanding maps with holes in [LiM].

\subsection{Relative compactness of pushed-forward densities}

What one hopes to do here is to identify a compact convex subset $\mathcal{K}$ of a suitable Banach space of functions with the property that (i) $\mathcal{P}_{1}^{n_{0}} 1 \in \mathcal{K}$ for some $n_{0} \geq 0$ and (ii) for all $f \in \mathcal{K}, \mathcal{P}_{1} f \in \mathcal{K}$. We then try to locate an a.c.c.i.m. in $\mathcal{K}$.

\section{Fixed point theorems}

We consider $\mathcal{P}_{1}: \mathcal{K} \rightarrow \mathcal{K}$, and seek to use, for example, the Schauder Fixed Point Theorem. A fixed point for $\mathcal{P}_{1}$ yields an a.c.c.i.m. Notice that no linearity is required of $\mathcal{P}_{1}$ (it is not linear); however, one has to make sure that it is defined and continuous on all of $\mathcal{K}$ (e.g. it is not defined for densities which are supported on finitely many pre-images of the hole).

Once a fixed point is identified, the following trick has been used to give uniqueness: Suppose that (i) there is a slightly larger set $\mathcal{D} \supset \mathcal{K}$ with the property that for all $g \in \mathcal{D}, \mathcal{P}_{1}^{n} g \rightarrow \mathcal{K}$, and (ii) all conditionally invariant densities in $\mathcal{K}$ have the same escape rate and are bounded away from 0 and $\infty$ with uniform bounds. Let $f_{0}$ and $f_{1}$ be two such densities. Consider $f_{s}=s f_{0}+(1-s) f_{1}, s$ not necessarily in $[0,1]$. Notice that $f_{s}$ is a legitimate conditionally invariant density as long as $f_{s} \geq 0$. A contradiction is reached if the family $\left\{f_{s}\right\}$ extends beyond $\mathcal{K}$ into $\mathcal{D}$.

This approach has been used to study expanding maps in $\mathbb{R}^{n}$ which admit a finite Markov partition after the introduction of holes [PY], piecewise expanding maps of the interval with generic small holes $[\mathrm{BC}]$, and Markov towers with holes [D1]. 


\section{Averaging techniques}

Averaging techniques for open systems are more difficult to implement because of the normalization: $\int \mathcal{P} g d m<\int g d m$ in general for $g \geq 0$, and $\mathcal{P}_{1}$ is not linear (so for example $\left.\mathcal{P}_{1}\left(\frac{1}{n} \sum_{i<n} \mathcal{P}_{1}^{i}(g)\right) \neq \frac{1}{n} \sum_{i \leq n} \mathcal{P}_{1}^{i+1}(g)\right)$. We outline below a version of the method proposed in [CMM1]:

Let $\alpha=\max \left\{r \in \mathbb{R}: \sum_{i=0}^{\infty} r^{i} m\left(M^{i}\right)<\infty\right\}<\infty$ and assume that $\sum_{i=0}^{\infty} \alpha^{i} m\left(M^{i}\right)=\infty .{ }^{4}$ Let

$$
f_{n}=\frac{\sum_{i=0}^{n} \alpha^{i} \mathcal{P}^{i} 1}{\sum_{i=0}^{n} \alpha^{i} m\left(M^{i}\right)} .
$$

The hope is to find $\mathcal{K}$ such that (i) $\mathcal{P}_{1}^{i} 1 \in \mathcal{K}$ for all $i$; (ii) linear combinations of $\mathcal{P}_{1}^{i} 1$ such as $f_{n}$ are also in $\mathcal{K}$; and (iii) if $f_{n} \rightarrow f$ say in $L^{1}$, then $f \in \mathcal{K}$. Let $\mu_{n}$ be the measure with density $f_{n}$, and $\mu$ a limit point of $\mu_{n}$ in the sense below. To see that $\mu$ is conditionally invariant, we write

$$
T_{*} \mu_{n}(A)=\frac{\sum_{i=0}^{n} \alpha^{i} T_{*}^{i+1} m(A)}{\sum_{i=0}^{n_{j}} \alpha^{i} m\left(M^{i}\right)}=\alpha^{-1} \mu_{n}(A)-\alpha^{-1} \frac{m(A)}{\sum_{i=0}^{n} \alpha^{i} m\left(M^{i}\right)}+\frac{\alpha^{n} T_{*}^{n+1} m}{\sum_{i=0}^{n} \alpha^{i} m\left(M^{i}\right)} .
$$

As $n \rightarrow \infty$, the second term vanishes since the series diverges. One expects in general the third term to vanish as well ${ }^{5}$ (notice that the series cannot diverge exponentially by our choice of $\alpha$ ). With both terms vanishing, it follows that $\mu$ is conditionally invariant with $\lambda=\alpha^{-1}$. Notice that this method does not assume that the escape rate of Lebesgue measure exists. In general, $\alpha^{-1}=\bar{\Lambda}$, which is associated with the slowest rate of escape.

Remark. Observe that the techniques presented in this subsection by themselves do not imply $\mathcal{T}_{1}^{n} m \rightarrow \mu$ where $\mu$ is any of the a.c.c.i.m. constructed. Such convergence, if valid, must be proved separately. This remark also applies to the construction of SRB measures for closed systems, in which the following two steps are often carried out separately: (1) One constructs first, by pushing forward Lebesgue measure on an unstable leaf and averaging as above, an invariant measure $\mu$ with smooth conditional measures on unstable manifolds; (2) one then deduces, using the absolute continuity of the stable foliation, that the set of points future-generic with respect to $\mu$ has positive Lebesgue measure. Some authors refer to measures $\mu$ with the property in (1) as "SRB measures" and those with the property in (2) as "physical measures"; see $[\mathrm{ER}]$ and [Y4]. For open systems, it is not clear what the notion analogous to "physical measure" should be: with respect to any conditionally invariant measure and possibly Lebesgue measure, the trajectory corresponding to almost every initial condition eventually escapes.

\subsection{Introducing Markov structures}

The following two sets of techniques have been used on systems that are uniformly expanding or hyperbolic, or predominantly so with mild singularities or discontinuities.

\section{Approximation by finite state Markov chains}

This method follows closely ideas developed by Sinai [S] for closed systems.

\footnotetext{
${ }^{4}$ If $\sum_{i=0}^{\infty} \alpha^{i} m\left(M^{i}\right)<\infty$, then one can introduce corrective factors to make the series diverge. See [CMM1] for details.

${ }^{5} f_{n}$ can be defined differently if this is a problem; see [CMM1]
} 
Suppose a hyperbolic map $\hat{T}: \hat{M} \circlearrowleft$ admits a finite Markov partition $\mathcal{R}=\left\{R_{1}, \ldots, R_{q}\right\}$. Consider first the case when $H=R_{q}$. We use the partition to code the system in the usual way and define the transition matrix $\Pi^{(0)}$ by

$$
\Pi_{i, j}^{(0)}=\frac{m\left(R_{i} \cap T^{-1} R_{j}\right)}{m\left(R_{i}\right)} \quad \text { for } i, j \leq q-1
$$

where as usual $T$ is the restricted map induced by $H$. Note that $\Pi^{(0)}$ is substochastic since entries involving $H$ are omitted. Assuming the usual aperiodic and irreducible conditions on $\Pi^{(0)}$, we obtain a unique probability vector, $p^{(0)}$, corresponding to the eigenvalue of largest magnitude, $\lambda^{(0)}$.

Refining the partition $\mathcal{R}$ by $\mathcal{R}^{(n)}=\bigvee_{i=-n}^{n} \hat{T}^{i}(\mathcal{R})$, we obtain a sequence of matrices, $\Pi^{(n)}$, which yield successively closer approximations to $T$ since $T$ is increasingly linear on elements of $\mathcal{R}^{(n)}$. Under suitable conditions, the sequence of probability vectors $p^{(n)}$ converges to an a.c.c.i.m. for $T$ with eigenvalue $\lambda=\lim _{n \rightarrow \infty} \lambda^{(n)}$. Control of the sequence $\left\{\Pi^{(n)}\right\}$ is typically achieved by first proving that there exists a unique family of invariant densities on the unstable leaves of the system which attracts all smooth conditional densities under the action of $\mathcal{P}_{1}$. This property implies that Lebesgue measure converges to this a.c.c.i.m. and that $\Lambda=\lambda$.

This method was first applied to Smale horseshoes in [C1] and [C2]. Later, it was applied to a simple class of billiards satisfying a fairly strong condition in [LM] and [Ri], and to Anosov diffeomorphisms in [CM1] and [CM2].

The case of small non-Markov holes for Anosov diffeomorphisms is studied in [CMT1] and [CMT2]. The authors approximate the hole $H$ by a sequence of holes $H_{k}$ made up of elements of the refined partitions $\mathcal{R}^{(k)}$. By the techniques of the previous paragraph, there is a natural a.c.c.i.m. $\mu_{k}$ corresponding to the open system associated with $H_{k}$. The weak limit of such measures is shown to be the natural a.c.c.i.m. for the original open system corresponding to the hole $H$.

\section{Countable Markov extensions}

For closed systems that are essentially uniformly hyperbolic except for the presence of certain recognizable structures that are obstructions to hyperbolicity, it has been demonstrated in many examples that countable Markov extensions capture quite effectively the statistical properties of the system (and are easier to construct than countable Markov partitions with similar control on recurrence times). By a Markov extension of a dynamical system $S: N \rightarrow N$, we refer to a tower map as described in Example 5, Sect. 4.2, for which $S$ is a quotient. See e.g. [Y2], [CY]. Because of the simple structure of tower maps, methods such as those in Sects. 5.2 and 5.3 apply. Once the pertinent results are obtained for the tower map, one seeks to pass them back to the original system.

In the context of open systems, Markov towers have been used to study piecewise expanding maps of the interval [D1] and logistic maps satisfying certain conditions [D2].

\section{$6 \quad$ Examples}

The purpose of this section is to see some of the ideas discussed in previous sections applied to two concrete settings.

Example 6. Piecewise expanding maps in one dimension. Let $\hat{T}:[0,1] \circlearrowleft$ be a $C^{2}$ piecewise expanding map satisfying $\left|\hat{T}^{\prime}\right| \geq \gamma>1$. 
Markov holes (special case). We assume $H$ is chosen so that $T: M^{1} \rightarrow M$ admits a finite Markov partition, and that some power of the transition matrix of the associated Markov chain is strictly positive.

Schauder Fixed Point Theorem: For a Lipschitz function $f$, we define the regularity of $f$ to be

$$
|f|_{r}=\sup \left\{\frac{\left|f^{\prime}(x)\right|}{f(x)}: f^{\prime}(x) \text { exists and } f(x) \neq 0\right\} \text {. }
$$

Let $\mathcal{D}=\left\{f \in C^{0}(M): f \geq 0\right.$ and $\left.|f|_{r}<\infty\right\}$ and let $\mathcal{K}_{B}=\left\{f \in \mathcal{D}:|f|_{r} \leq B, \int_{M} f d m=1\right\}$. For $B$ large enough, it is shown in $[\mathrm{PY}]$ that $\mathcal{P}_{1}\left(\mathcal{K}_{B}\right) \subset \mathcal{K}_{B}$, and for all $g \in \mathcal{D}, \lim _{n} \mathcal{P}_{1}^{n} g \rightarrow \mathcal{K}_{B}$. Since $\mathcal{K}_{B}$ is a convex compact subset of $C^{0}(M)$, the Schauder Fixed Point Theorem yields an a.c.c.i.m. with density $f \in \mathcal{K}_{B}$; uniqueness of this measure in $\mathcal{D}$ follows from the trick described in Sect. 5.3. The convergence of functions in $\mathcal{D}$ to $f$ requires a separate argument. See $[\mathrm{PY}]$ for details.

Projective cones: Let $\mathcal{C}_{B}=\left\{f \in D:|f|_{r} \leq B\right\}$. It is straightforward to verify that $\mathcal{C}_{B}$ is a convex cone in $C^{0}(M), \mathcal{P}\left(\mathcal{C}_{B}\right) \subset \mathcal{C}_{B}$, and the $L^{1}$ norm is adapted. Using the transitivity assumption, one can show that for any $g \in \mathcal{C}_{B}, \mathcal{P}^{N} g$ is bounded away from zero and infinity on $M$ for some fixed $N$ which does not depend on $g$. This in turn implies the finiteness of the diameter of $\mathcal{P}^{N}\left(\mathcal{C}_{B}\right)$ in the Hilbert metric. We conclude that there exists a unique $f \in \mathcal{C}_{B}$ which is a fixed point for $\mathcal{P}_{1}^{N}$. Since $f$ is unique, it is also a fixed point for $\mathcal{P}_{1}$ and is the limit in $L^{1}$ of all $g \in \mathcal{C}_{B}$. Finally, $1 \in \mathcal{C}_{B}$, so that the escape rate of Lebesgue measure exists and is given by that of this a.c.c.i.m.

The general case. Let $\mathcal{B}$ denote the set of functions on $[0,1]$ of bounded variation.

Schauder Fixed Point Theorem and Lasota-Yorke inequality: In [BC], the authors prove a LasotaYorke inequality for the unnormalized operator $\mathcal{P}$, namely

$$
\left\|\mathcal{P}^{n} f\right\| \leq C \sigma^{n}\|f\|+B|f|_{1}
$$

where $\|\cdot\|$ is the bounded variation norm and $|\cdot|_{1}$ is the $L^{1}$-norm. If the holes are sufficiently small, (8) implies that an iterate $N$ of the normalized operator $\mathcal{P}_{1}$ leaves the set $\mathcal{K}_{b}=\{f \in \mathcal{B}$ : $\left.\|f\| \leq b,|f|_{1}=1\right\}$ invariant, where $b$ is large enough that $C \sigma^{N} b+B \leq b$, but small enough that $\mathcal{K}_{b}$ does not include any functions supported entirely on the pre-image of the hole. The Schauder theorem applies to $\mathcal{P}_{1}$ acting on $\mathcal{K}_{b}$; as does the uniqueness argument in Sect. 5.3. Under an additional genericity assumption on the placement of the holes, all functions in $\mathcal{K}_{b}$ converge to $f$, so that $f$ defines a natural a.c.c.i.m.

Quasi-compactness of $\mathcal{P}$ : This is also implied by (8) coupled with the estimate $\left|\mathcal{P}^{n} f\right|_{1} \leq|f|_{1}$ and the fact that the unit ball of $\mathcal{B}$ is compact in $L^{1}$. In $[\mathrm{LiM}]$, the authors derived a similar inequality and showed that as operators from $\mathcal{B}$ to $L^{1}, \mathcal{P}_{T}$ and $\mathcal{P}_{\hat{T}}$ are close for small holes. More precisely, let $H_{\varepsilon}$ be a hole of size $\varepsilon$ and let $M_{\varepsilon}=\hat{M} \backslash H_{\varepsilon}$ with the analogous definitions for $M_{\varepsilon}^{n}$. If $\mathcal{P}_{\varepsilon}$ denotes the transfer operator for the open system with hole $H_{\varepsilon}$ and $\mathcal{P}_{0}=\mathcal{P}_{\hat{T}}$, then for any $f \in \mathcal{B}$,

$$
\int_{\hat{M}}\left|\mathcal{P}_{\varepsilon} f-\mathcal{P}_{0} f\right| d m \leq \int_{\hat{M}}\left|\mathcal{P}_{0}\left(1_{\hat{M} \backslash M_{\varepsilon}^{1}} f\right)\right| d m \leq \int_{\hat{M} \backslash M_{\varepsilon}^{1}}|f| d m \leq\|f\| m\left(\hat{M} \backslash M_{\varepsilon}^{1}\right) .
$$

It is clear that the last expression tends to zero with $\varepsilon$. Remark 5 of [KL] then implies that the spectra of $\mathcal{P}_{\varepsilon}$ and $\mathcal{P}_{0}$ differ by no more than a constant times $\varepsilon|\log \varepsilon|$. Thus if $\hat{T}$ has a 
unique invariant measure, then for sufficiently small holes, $T$ has a unique probability density $f \in \mathcal{B}$ corresponding to a unique eigenvalue of maximum modulus which is in fact $\Lambda$, and all the conclusions of Sect. 5.2 apply.

Remark. In some situations, it is possible to obtain a sharper bound on the perturbed eigenvalue. Let $\lambda_{\varepsilon}$ denote the eigenvalue of maximum modulus of $\mathcal{P}_{\varepsilon}$. Then for both expanding maps of the interval and Misiurewicz maps of the logistic family, it is shown in [D1] and [D2] using Markov extensions that $1-\lambda_{\varepsilon} \leq C \varepsilon$, so that the eigenvalue exhibits a Lipschitz dependence on the size of the hole.

In the example of the tent map from Sect. 3.2, observe the relation between the 3 conditionally invariant densities constructed there and the decomposition $\mathcal{B}=\mathcal{H} \oplus\{f\}$ in Sect. 5.2: The density in (i) is $f$, the one in (ii) is not in $\mathcal{B}$, and the family in (iii) is contained in $\mathcal{H}$.

Example 7. Linear toral automorphisms. Let $\hat{T}: \mathbb{T}^{2} \circlearrowleft$ be a hyperbolic linear toral automorphism, and let $\mathcal{R}=\left\{R_{1}, R_{2}, \cdots, R_{q}\right\}$ be a Markov partition whose elements are (real) rectangles. Let $H=R_{q}$. In this simple example, the convergence of $\mathcal{T}_{1}^{n} m$, the geometry of the resulting a.c.c.i.m. and its escape rate are all quite transparent as we now demonstrate.

We fix $k, 1 \leq k \leq q-1$, and show first that $\left(\mathcal{T}_{1}^{n} m\right)\left(R_{k}\right)$ converges as $n \rightarrow \infty$. Let $\hat{Q}=\left(q_{i j}\right)$ be the matrix corresponding to the topological Markov shift defined by $\hat{T}$, i.e. $q_{i j}=1$ if and only if $m\left(R_{i} \cap \hat{T}^{-1} R_{j}\right)>0,=0$ otherwise; and let $Q$ be the $(q-1) \times(q-1)$ matrix obtained by deleting the $q$ th row and $q$ th column of $\hat{Q}$. We say an $(n+1)$-block $\left(i_{0}, i_{1}, \cdots, i_{n}\right), i_{j} \in\{1,2, \cdots q-1\}$, is admissible if $q_{i_{j} i_{j+1}}=1$ for $j=0, \cdots, n-1$. Then

$$
\left(\mathcal{T}_{1}^{n} m\right)\left(R_{k}\right)=\frac{\sum_{i=1}^{q-1} N_{i k}^{n} \cdot\left|R_{i}^{s}\right| \alpha^{-n}\left|R_{k}^{u}\right|}{\sum_{i, j=1}^{q-1} N_{i j}^{n} \cdot\left|R_{i}^{s}\right| \alpha^{-n}\left|R_{j}^{u}\right|}
$$

where $N_{i j}^{n}$ is the number of admissible $(n+1)$-blocks starting in $i$ and ending in $j,\left|R_{i}^{s}\right|$ is the length of the stable segments in $R_{i},\left|R_{i}^{u}\right|$ is the length of the unstable segments in $R_{i}$, and $\alpha>1$ is the derivative of $\hat{T}$ in the unstable direction. Note that $\left|R_{i}^{s}\right| \alpha^{-n}\left|R_{j}^{u}\right|$ is the area of the subrectangle in $R_{i}$ corresponding to any one of the $(n+1)$-blocks starting in $i$ and ending in $j$.

Now $N_{i j}^{n}=\left(Q^{n}\right)_{i j}$, the $(i, j)$-entry of $Q^{n}$. Assuming $Q$ is irreducible and aperiodic, the columns of $Q^{n}$, multiplied by $\beta^{-n}$ where $\beta$ is the largest eigenvalue of $Q$, converges exponentially to the right eigenvector of $Q$. It follows that

$$
\left(\mathcal{T}_{1}^{n} m\right)\left(R_{k}\right) \rightarrow \frac{\left|R_{k}^{u}\right|}{\sum_{j=1}^{q-1}\left|R_{j}^{u}\right|} \quad \text { as } \mathrm{n} \rightarrow \infty .
$$

The convergence of $\left(\mathcal{T}_{1}^{n} m\right)(A)$ where $A$ is a subrectangle of $R_{k}$ obtained by fixing its itinerary in the last $\ell$ iterates is computed similarly. This proves that $\mathcal{T}_{1}^{n} m \rightarrow \mu$ where on each $R_{k}, \mu$ is the product of Lebesgue measure on unstable segments and a measure supported on a Cantor set in the stable direction.

It remains to estimate the escape rate $-\log \Lambda$. The same reasoning as before gives

$$
\frac{m\left(M^{n+1}\right)}{m\left(M^{n}\right)}=\frac{\sum_{i, j=1}^{q-1} N_{i j}^{n+1} \cdot\left|R_{i}^{s}\right| \alpha^{-(n+1)}\left|R_{j}^{u}\right|}{\sum_{i, j=1}^{q-1} N_{i j}^{n} \cdot\left|R_{i}^{s}\right| \alpha^{-n}\left|R_{j}^{u}\right|} \rightarrow \beta \alpha^{-1}=: \Lambda \quad \text { as } \mathrm{n} \rightarrow \infty .
$$

Notice that $\log \beta$ is the topological entropy of the Markov shift defined by $Q$, or equivalently, the topological entropy of $\hat{T}$ restricted to the set $\Omega:=\cap_{n \in \mathbb{Z}} \hat{T}^{n}(M)$. Here we see clearly why $\log \Lambda=$ topological entropy - positive Lyapunov exponent, which in this linear case is equal to topological pressure as asserted in (5) in Sect. 4.1. 


\section{More on Relation to SRB Measures}

Numerous relations to SRB measures have been encountered in previous sections. We finish by posing a few questions or conjectures that further amplify these connections.

\section{A. What is a natural conditionally invariant measure? (Summary of discussion)}

We think of a conditionally invariant measure $\mu$ as natural if $\mathcal{T}_{1}^{n} m \rightarrow \mu$ as $n \rightarrow \infty$. A weaker requirement is that $\mu$ is a limit point of averages of these pushed-forward measures in the sense of Section 5. Since $T_{*}^{n} m$ is the image under $T_{*}^{n}$ of Lebesgue measure restricted to $M^{n}$, one expects $\mathcal{T}_{1}^{n} m$ to have a density (or a density on unstable manifolds) if $\left.T^{n}\right|_{M^{n}}$ is sufficiently expanding (or sufficiently expanding on unstable manifolds). If these densities lie in a compact set, their properties are passed on to $\mu$. Thus we see that the idea is the same as in the construction of SRB measures, except that we consider the forward images of Lebesgue measure on $M^{n}$, not on all of $\hat{M}$.

The measures in the last paragraph are to be distinguished from those constructed by starting with some arbitrary density on $M \backslash M^{1}$ and pulling it backwards. As shown in Sect. 3.1, this can lead to well-defined a.c.c.i.m. with overlapping supports that are completely arbitrary and not necessarily representative of the escape dynamics starting from Lebesgue measure. The presence of this (mostly meaningless) class of a.c.c.i.m. is an important difference between SRB measures and natural conditionally invariant measures; it is also the underlying cause for the difficulties in interpreting certain existence results.

Questions: Under what conditions do natural conditionally invariant measures exist? Specifically, if $\hat{T}$ is nonuniformly expanding and has an invariant density, or if it has a nonuniformly hyperbolic attractor with an SRB measure, should one expect it to have a natural a.c.c.i.m. on reasonable regions $M$ of the phase space?

Known results are limited mostly to the uniformly expanding and hyperbolic systems mentioned previously; the only genuinely nonuniform situation studied so far is [D2].

\section{B. Stability of SRB measures with respect to "leaks"}

A relevant notion of stability for an SRB measure is that it is not unduly altered by the presence of small leaks in the system. More precisely, for a sequence of holes $\left\{H_{\varepsilon}, \varepsilon>0\right\}$, we assume there exist natural conditionally invariant measures $\mu_{\varepsilon}$ for the open systems on $M_{\varepsilon}=\hat{M} \backslash H_{\varepsilon}$. We say an SRB measure $\mu_{0}$ is stable with respect to leaks if for every sequence of holes $\left\{H_{\varepsilon}\right\}$ with reasonable properties and tending to zero in diameter, we have $\mu_{\varepsilon} \rightarrow \mu_{0}$ weakly as $\varepsilon \rightarrow 0$.

Questions: Under what conditions are SRB measures stable with respect to leaks? Among the closed systems known to have SRB measures, which ones have this property? What about billiards, our motivating example?

Affirmative answers to this question have been given for Anosov and certain one-dimensional maps; see [CMT1], [LiM], [D1], [HY] and [D2]. In relation to the stability property above, we have the following general observation:

Lemma 7.1 Let $\hat{T}$ be a piecewise differentiable map on a Riemannian manifold $\hat{M}$ and let $m$ be the Riemannian volume. Let $\left\{H_{\varepsilon}\right\}$ be a sequence of holes satisfying $m H_{\varepsilon}=\varepsilon$ and define the usual restricted maps $T_{\varepsilon}$ and manifolds $M_{\varepsilon}$. Assume that as $n \rightarrow \infty$, 
1. $\hat{T}_{*}^{n} m \rightarrow \mu_{0}$ where $\mu_{0}$ is the SRB measure for $\hat{T}$ and

2. $T_{\varepsilon *}^{n} m / T_{\varepsilon *}^{n} m\left(M_{\varepsilon}\right) \rightarrow \mu_{\varepsilon}$ uniformly in $\varepsilon$;

then $\mu_{\varepsilon} \rightarrow \mu_{0}$ as $\varepsilon \rightarrow 0$.

Proof. For any Borel set $A$ and $n \geq 0$,

$$
\left|\mu_{0}(A)-\mu_{\varepsilon}(A)\right| \leq\left|\mu_{0}(A)-\hat{T}_{*}^{n} m(A)\right|+\left|\hat{T}_{*}^{n} m(A)-\frac{T_{\varepsilon *}^{n} m(A)}{T_{\varepsilon *}^{n} m\left(M_{\varepsilon}\right)}\right|+\left|\frac{T_{\varepsilon *}^{n} m(A)}{T_{\varepsilon *}^{n} m\left(M_{\varepsilon}\right)}-\mu_{\varepsilon}(A)\right| .
$$

For any $\delta>0$, choose $N$ independent of $\varepsilon$ such that the first and third terms above are less than $\delta$. For this fixed $N$, choose $\varepsilon$ so small that the second term is less than $\delta$.

\section{Geometric property of natural conditionally invariant measures}

Our next question asks if natural conditionally invariant measures have the same geometric characterization as SRB measures. Note that the entire backward orbit of $\mu$-a.e. $x \in M$ lies in $M$, so unstable manifolds can potentially be defined.

Questions: Assume $\hat{T}$ is invertible, $M$ is a reasonable region, and $\mathcal{T}_{1}^{n} m \rightarrow \mu$. Are Lyapunov exponents well defined $\mu$-a.e.? If so, and if at least one of the Lyapunov exponents is positive $\mu$ a.e., does it follow that $\mu$ has absolutely continuous conditional measures on unstable manifolds?

The Lyapunov exponents question has not been addressed in the literature. Absolute continuity of conditional measures on unstable manifolds has been established for the uniformly hyperbolic systems mentioned earlier [C1], [LM], [Ri], [CM1], [CM2], [CMT1].

\section{Invariant measures associated with a.c.c.i.m. and their escape rates}

Let $\mu$ be the natural a.c.c.i.m. and $\lambda$ its escape rate. For each $n$, define

$$
\mu_{n}(A)=\lambda^{-n} \mu\left(A \cap M^{n}\right)
$$

for any Borel subset $A$ of $M$. Since $\hat{T}_{*} \mu_{n}(A)=\mu_{n-1}(A), \mu_{n}$ converges as $n \rightarrow \infty$ to a $\hat{T}$ invariant probability measure $\nu$ supported on the invariant set $\Omega=\cap_{n \in \mathbb{Z}} \hat{T}^{n} M$. For uniformly hyperbolic systems, it has been shown that $\nu$ is the equilibrium state of the potential $\varphi=$ $-\log \left|\operatorname{det}\left(\left.D \hat{T}\right|_{E^{u}}\right)\right|$ on $\Omega$ [C1], [CMS1], [CMS2], [LM], [Ri], [CM1], [CMT2]. This implies in particular that $\log \Lambda=h_{\nu}+\int \varphi d \nu$; see Sect. 4.1 .

Question: Is this true in greater generality, with $\varphi$ equal to the sum of positive Lyapunov exponents? For example, does it carry over to systems which admit Markov extensions?

\section{References}

[BBS] V. Baladi, C. Bonatti and B. Schmitt, Abnormal escape rates from nonuniformly hyperbolic sets, Ergod. Th. Dynam. Sys. 19:5 (1999), 1111-1125.

[BK] V. Baladi and G. Keller, Zeta functions and transfer operators for piecewise monotonic transformations, Comm. Math. Phys. 127 (1990), 459-477. Lecture Notes in Mathematics, 470. Springer-Verlag: Berlin, 1975.

[BC] H. van den Bedem and N. Chernov, Expanding maps of an interval with holes, Ergod. Th. and Dynam. Sys. 22 (2002), 637-654. 
[Bi] G. Birkhoff, Lattice Theory, $253^{\text {rd }}$ ed. A.M.S. Colloq. Publ., Providence, Rhode Island (1967).

[BB] M. Blank and L. Bunimovich, Multicomponent dynamical systems: SRB measures and phase transitions, Nonlinearity 16 (2003), 387-401.

[B] R. Bowen, Equilibrium states and the ergodic theory of Anosov diffeomorphisms. Lecture Notes in Mathematics, 470. Springer-Verlag: Berlin, 1975.

[C1] N. N. Čencova, A natural invariant measure on Smale's horseshoe, Soviet Math. Dokl. 23 (1981), 87-91.

[C2] N. N. Čencova, Statistical properties of smooth Smale horseshoes, in Mathematical Problems of Statistical Mechanics and Dynamics, R. L. Dobrushin, ed. Reidel: Dordrecht, 1986, pp. 199-256.

[CM1] N. Chernov and R. Markarian, Ergodic properties of Anosov maps with rectangular holes, Bol. Soc. Bras. Mat. 28 (1997), 271-314.

[CM2] N. Chernov and R. Markarian, Anosov maps with rectangular holes. Nonergodic cases, Bol. Soc. Bras. Mat. 28 (1997), 315-342.

[CMM1] P. Collet, S. Martínez and V. Maume-Deschamps, On the existence of conditionally invariant probability measures in dynamical systems, Nonlinearity 13 (2000), 12631274 .

[CMM2] P. Collet, S. Martínez and V. Maume-Deschamps, Corrigendum: On the existence of conditionally invariant probability measures in dynamical systems, Nonlinearity $\mathbf{1 7}$ (2004) 1985-1987.

[CMS1] P. Collet, S. Martínez and B. Schmitt, The Yorke-Pianigiani measure and the asymptotic law on the limit Cantor set of expanding systems, Nonlinearity 7 (1994), 14371443.

[CMS2] P. Collet, S. Martínez and B. Schmitt, Quasi-stationary distribution and Gibbs measure of expanding systems, in Instabilities and Nonequilibrium Structures V, E. Tirapegui and W. Zeller, eds. Kluwer: Dordrecht, 1996, pp. 205-19.

[CMS3] P. Collet, S. Martínez and B. Schmitt, The Pianigiani-Yorke measure for topological Markov chains, Israel J. of Math. 97 (1997), 61-70.

[CMS4] P. Collet, S. Martínez and B. Schmitt, On the enhancement of diffusion by chaos, escape rates and stochastic stability, Trans. Amer. Math. Soc. 351:7 (1999), 2875-2897.

[CMT1] N. Chernov, R. Markarian and S. Troubetskoy, Conditionally invariant measures for Anosov maps with small holes, Ergod. Th. and Dynam. Sys. 18 (1998), 1049-1073.

[CMT2] N. Chernov, R. Markarian and S. Troubetskoy, Invariant measures for Anosov maps with small holes, Ergod. Th. and Dynam. Sys. 20 (2000), 1007-1044.

[CY] N. Chernov and L.-S. Young, Decay of correlations for Lorenz gases and hard balls, in Hard Ball Systems and the Lorenz Gas, D. Szasz, ed., Encyclopaedia of Mathematical Sciences 101. Springer-Verlag: Berlin, 2000, pp. 89-120. 
[D1] M. Demers, Markov Extensions for Dynamical Systems with Holes: An Application to Expanding Maps of the Interval, Israel J. of Math. 146 (2005), 189-221.

[D2] M. Demers, Markov Extensions and Conditionally Invariant Measures for Certain Logistic Maps with Small Holes, Ergod. Th. and Dynam. Sys. 25:4 (2005), 1139-1171.

[ER] J.-P. Eckmann and D. Ruelle, Ergodic theory of chaos and strange attractors, Rev. Modern Phys. 57:3 (1985), 617-656.

[FKMP] P.A. Ferrari, H. Kesten, S. Martínez, and P. Picco, Existence of quasi-stationary distributions. A renewal dynamical approach, Annals of Prob. 23:2 (1995), 501-521.

[FS] P. Ferrero and B. Schmitt, Ruelle's Peron-Frobenius theorem and projective metrics, Coll. Math. Soc. J. Bollyai, 27 (1979).

[HY] A. Homburg and T. Young, Intermittency in families of unimodal maps, Ergod. Th. and Dynam. Sys. 22:1 (2002), 203-225.

[KL] G. Keller and C. Liverani, Stability of the spectrum for transfer operators, Ann. Scuola Norm. Sup. Pisa Cl. Sci. (4) 28 (1999), 141-152.

[L] C. Liverani, Decay of Correlations, Ann. Math. 142:2 (1995), 239-301.

[LiM] C. Liverani and V. Maume-Deschamps, Lasota-Yorke maps with holes: conditionally invariant probability measures and invariant probability measures on the survivor set, Annales de l'Institut Henri Poincaré Probability and Statistics, 39 (2003), 385-412.

[LM] A. Lopes and R. Markarian, Open Billiards: Cantor sets, invariant and conditionally invariant probabilities, SIAM J. Appl. Math. 56 (1996), 651-680.

[PY] G. Pianigiani and J. Yorke, Expanding maps on sets which are almost invariant: decay and chaos, Trans. Amer. Math. Soc. 252 (1979), 351-366.

[Ri] P.A. Richardson, Jr., Natural Measures on the Unstable and Invariant Manifolds of Open Billiard Dynamical Systems, Doctoral Dissertation, Department of Mathematics, University of North Texas, 1999.

[S] Y. Sinai, Gibbs measures in ergodic theory, Russ. Math. Surveys, 27 (1972) 21-69.

[V] D. Vere-Jones, Geometric ergodicity in denumerable Markov chains, Quart. J. Math. 13 (1962), 7-28.

[Y1] L.-S. Young, Some large deviation results for dynamical systems, Trans. Amer. Math. Soc. 318 (1990), 525-543.

[Y2] L.-S. Young, Statistical properties of dynamical systems with some hyperbolicity, Ann. Math. 147 (1998), 585-650.

[Y3] L.-S. Young, Recurrence times and rates of mixing, Israel J. of Math. 110 (1999), 153-188.

[Y4] L.-S. Young, What are SRB measures and which dynamical systems have them? J. Stat. Phys. 108 (2002), 733-754. 